\title{
Dynamic Impedance Functions of a Square Foundation Estimated with an Equivalent Linear Approach
}

\author{
Badreddine Sbartai ${ }^{1,2(\bowtie)}$ \\ 1 Université Badji Mokhtar, Annaba, Algeria \\ bsbartai@hotmail.fr \\ ${ }^{2}$ LMGHU, Université 20 Août 1955, Skikda, Algeria
}

\begin{abstract}
This paper presents the role of the intensity of an earthquake on the estimation of dynamic impedance functions. For various seismic shaking we will have different nonlinear behavior of the soil, which may have significant effects on the amplitude and shape of the dynamic stiffness and damping coefficient. However, under the assumption of linear elastic behavior of the soil, loss of soil stiffness under strong shaking is not taken into account in the dynamic impedance functions. The dynamic impedance functions are estimated using an equivalent linear process and compared relative to the linear elastic case. The vibrations originate from the rigid foundation embedded in the soil layer, which are subjected to harmonic loads of translation, rocking, and torsion. The dynamic responses of the rigid surface foundation are solved from the wave equations by taking into account their interaction. The solution is formulated using the frequency domain boundary element method (BEM), in conjunction with Kausel-Peek Green's function for a layered stratum and the thin layer method (TLM) to account for the interaction between the soil-foundation. A parametric analysis is performed for surface foundation in a semi-infinite soil limited by bedrock and subjected to three earthquake records.
\end{abstract}

\section{Introduction}

The analysis of the behavior of foundations under dynamic loads has grown considerably over the past four decades. The stringent security requirements imposed on design of certain types of structures have played a particularly important role in the development of analytical and numerical methods. The key step in studying the dynamic response of foundations is the determination of the relationship between forces and displacements. This relationship is expressed using impedance functions (dynamic stiffness) or the compliance functions (dynamic flexibility). The consideration of the soil-structure interaction in the analysis of the dynamic behavior of foundations allows taking realistically into account the influence of soil on its vibration. Several methods have been proposed in the literature to solve the soil-structure interaction problem. To simplify the problem, linear-analysis techniques have been developed. One of the most commonly used approaches is the substructures method that allows the problem to be analyzed in two parts Kausel et al., Aubrey et al. and Pecker. In this approach, the dynamic responses of superstructure and of the 
substructure are examined separately. The analysis of foundations systems can be reduced to the study of the dynamic stiffness at the soil-foundations interface (known as impedance function). Although a solution of a soil-structure interaction problem in most cases involves a straightforward application of any of the well-established soil-structure interaction methods, a relatively small number of 3-D investigations have appeared in the related literature. This is probably due to the substantial computational effort required by the Finite Element Method (FEM) and the Boundary Element Method (BEM). Furthermore, there is a noticeable absence of simplified discrete models, which is due, perhaps, to lack of rigorous results that could be used for the verification and calibration of such models. The complexities of the shapes of foundations, of the loadings, and of the soil conditions have discouraged, in general, the development of analytical solutions.

In the nonlinear case, the behavior of dynamically loaded foundations and the soil layers were investigated to a lesser degree (Borja and Wu 1994). For this, we simulate numerically the dynamic response of a massless square foundation resting on a semi-infinite soil considering equivalent-linear soil behavior described through strain-compatible shear modulus and damping coefficients for a suite of earthquake records. The method is based on subdivision of the soil mass under the foundation into a number of horizontal layers of different shear modulus and damping ratio like thin layer method (TLM) but compatible with the level of strain imposed by an earthquake motion or a dynamic load. Indeed, the semi-infinite soil is replaced by a layered profile with strain-compatible properties within each layer.

In this study, the dynamic responses of the rigid surface foundation are solved from the wave equations by taking into account their interaction. The solution is formulated using the frequency domain boundary element method (BEM), in conjunction with Kausel-Peek Green's function for a layered stratum and the thin layer method (TLM) to account for the interaction between the soil-foundation (Sbartai and Boumekik 2008), Sbartai (2016) and Messioud et al. (2016). For a given set of applied loads, characteristic strains are determined in each soil layer and the analysis is repeated in an iterative manner until convergence in material properties is achieved. For limited space, a parametric analysis is performed only for square foundation resting on a semi-infinite soil excited by a suite of three recorded earthquakes motions. The dimensionless results are provided for the variation of foundation stiffness and damping with frequency and excitation level in vertical, horizontal, rocking, and torsional modes.

\subsection{Calculation Model}

There are two main methods dealing the soil-structure interaction analysis: direct method, and of the sub-structure method. In the direct method, the response of the soil and structure is determined simultaneously by analysing the idealized soil-structure system in a single step. In substructure method, the soil-structure interaction problem is divided into two sets of simpler problems, which are solved independently, and the results are then superposed to obtain the response of the structure. The basic step in the substructure approach is to determine the force-displacement characteristics of the soil. This relationship may be in the form of an impedance (stiffness) function, or, inversely, 
a compliance (flexibility) function. By definition, the impedance $\mathrm{K}(6,6)$ of the system is the relation between the load $\mathrm{P}(6,1)$ and the response $\mathrm{U}(6,1)$. Generally, the load, the impedance and the response are complex quantities. The relationship between impedance, displacement and applied load is given by

$$
P(\omega)=K(\omega) \cdot U(\omega)
$$

where $K(\omega)$ is a symmetric matrix $(6,6)$ given by

$$
K(\omega)=\left[\begin{array}{cccccc}
K_{11}(\omega) & 0 & 0 & 0 & K_{1,5}(\omega) & 0 \\
0 & K_{22}(\omega) & 0 & K_{2,4}(\omega) & 0 & 0 \\
0 & 0 & K_{33}(\omega) & 0 & 0 & 0 \\
0 & K_{4,2}(\omega) & 0 & K_{44}(\omega) & 0 & 0 \\
K_{5,1}(\omega) & 0 & 0 & 0 & K_{55}(\omega) & 0 \\
0 & 0 & 0 & 0 & 0 & K_{66}(\omega)
\end{array}\right]
$$

In the particular case of a symmetric surface foundation (i.e., rectangular, square), it is possible to uncouple the impedance matrix along the two principal axes ( $\mathrm{x}$ and $\mathrm{y}$ ) to reduce its dimension (i.e., $K_{51}=K_{15}=K_{42}=K_{24}=0$ ).

The model of calculation is represented in Fig. 1. The considered foundation is rigid, square shape, and placed on the surface or partially embedded in homogeneous (heterogeneous) soil that is limited by a semi-infinite soil. The soil is viscoelastic linear, as characterized by its mass density $\rho$, shear modulus $G$, damping coefficient $\beta$, and Poisson's ratio $v$. The foundations are subjected to three harmonic external forces $-\mathrm{P}_{\mathrm{x}}, \mathrm{P}_{\mathrm{y}}$, and $\mathrm{P}_{\mathrm{z}}$ at three harmonic moments- $-\mathrm{M}_{\mathrm{x}}, \mathrm{M}_{\mathrm{y}}$, and $\mathrm{M}_{\mathrm{z}}$. It is assumed that the time dependence of the excitation is of type $\mathrm{e}^{\mathrm{i} \omega \mathrm{t}}$, in which $\omega$ denotes the frequency. For brevity, this time factor will be omitted. The goal is to obtain the impedance functions.

Displacements in an unspecified point " $\alpha$ " of the soil may be obtained from the wave equation solution

$$
\left(\left(C_{p}^{2}-C_{s}^{2}\right) u_{j, i j}+C_{s}^{2} u_{i, j j}+C_{p}^{2} \omega^{2} u_{i}\right)_{\rho}=0
$$

where $u_{i}$ is the $\mathrm{x}$-component of harmonic displacement amplitude vector; $u_{j}$ is the $\mathrm{y}$-component of the harmonic displacement amplitude vector; $u_{j, i j}$ is the partial derivative of $u_{j}$ with respect to the $\mathrm{x}$ - and y-axes; $u_{i, j j}$ is the second partial derivative of $u_{i}$ with respect to the y-axis; $C_{s}$ and $C_{p}$ are the shear (S)- and compression (P)-wave velocities; $\rho$ is the mass density; and $\omega$ is the angular frequency of excitation.

The solution of Eq. (3) can be obtained in the form of the following boundary integral equation in the frequency domain

$$
u_{j}(x, \omega)=\int_{s} G_{i j}(x, \xi, \omega) \cdot t_{i}(\xi, \omega) \cdot d S(\xi) .
$$




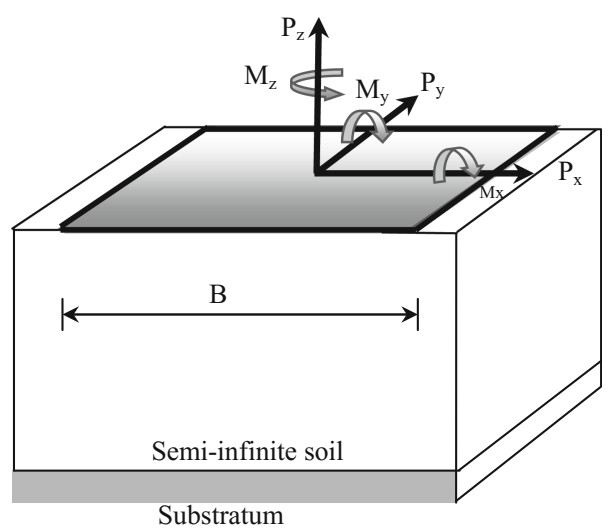

(a)

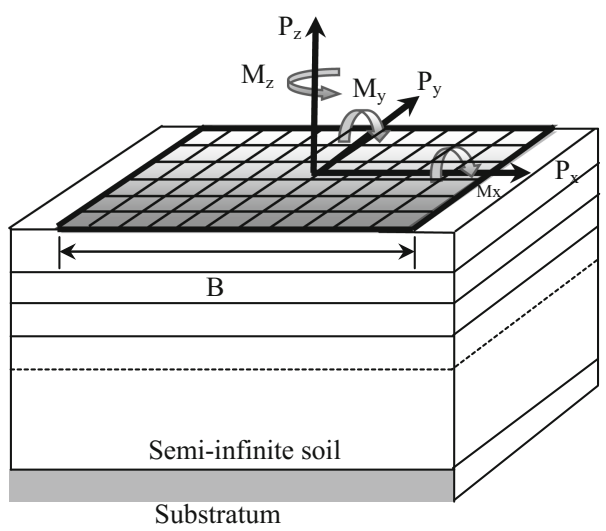

(b)

Fig. 1. (a) Geometric model (b) discretized model.

Where $G_{i j}$, represents Green's functions tensor and $t_{i}$ is the unknown surface traction.

Equation (4) remains to be solved as long as the domain is a continuum. However, if the domain is discretized in an appropriate form, Eq. (4) can be algebraically evaluated for each element. In this approach, the discretization principle of the soil mass, shown in Fig. 2, is based on two types of discretization, one horizontal and one vertical. The horizontal discretization consists of subdividing any horizontal section of the soil mass into square elements. The average displacement of the element is replaced by its center displacements, in which the distribution of constraints is supposed to be uniform. The vertical discretization consists of subdividing the solid mass of the soil in the under layers (infinite elements in the horizontal direction), which have rather low thicknesses compared with the Rayleigh wavelength $(\lambda / 10)$, to linearized the 

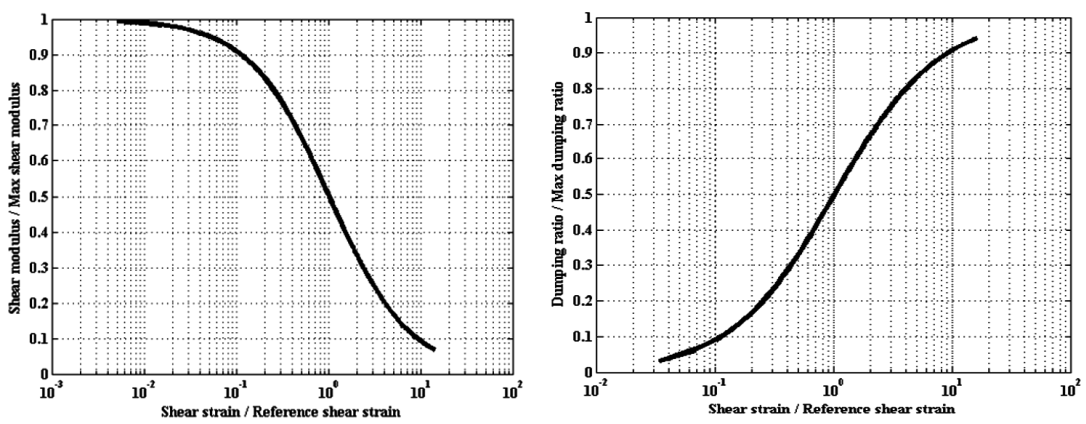

Fig. 2. Shear modulus reduction and damping increase curves

displacement of one under layer to the other. In the discretized model, Eq. (4) is expressed in algebraic form as follows

$$
u_{j}=\sum_{i=1}^{N R T} \int_{s} G_{i j} \cdot t_{i} \cdot d S,
$$

where NRT represents the total number of elements that discretized the free surface and the interface between the soil and the foundation.

The Green's functions thus obtained are complex and constitute the starting point for the determination of the flexibility matrix of a volume of the arbitrary soil. For more details about the computation of the above Green's functions, one can consult the work of (Kausel and Peek 1982) and Boumekik doctoral dissertation Boumekik (1985). Viscoelastic soil behavior can be easily introduced in the present formulation by simply replacing the elastic constants $\lambda$ and $G$ with their complex values

$$
\begin{aligned}
\lambda * & =\lambda(1+2 i \beta) \\
G * & =G(1+2 i \beta),
\end{aligned}
$$

where, $\beta$ is the hysteretic damping coefficient.

The equivalent linear analysis has been used to described the non-linear behavior of the soil through strain-compatible shear modulus and damping coefficients for a suite of earthquake records. Assuming that the substratum and the interfaces between the different layers of soil are essentially horizontal, we can consider each layer as linear elastic and develop a model with lumped mass in order to analyze the nonlinear dynamic response of the soil deposit during an earthquake. Thus, Kryloff and Bogoliuboff (1943) and Bogoliuboff and Mitropolsky (1961) proposed the use of both an equivalent linear spring constant, and an equivalent damping ratio for a single-degree-of-freedom system having nonlinear characteristics. Indeed, (Seed and Idriss, 1969) have proposed the use of an equivalent linear scheme wherein the shear modulus and damping are modeled using a linear spring and a dashpot, respectively. The parameters of the spring and the dashpot are calculated based on the secant shear 
modulus and damping ratio for a given level of shear strain. Shear modulus and damping ratio values are iteratively calculated based on the computed strain. For earthquake input motion, (Seed and Idriss 2012) suggested that the properties must be calculated for a strain equal to $2 / 3$ of maximum strain level in a given layer.

The knowledge of Green's functions allows obtaining harmonic displacements resulting from the discretized domain by successive application of unit forces on all the interface elements, and thus flexibility matrix is constructed. In the particular case of a symmetric foundation (i.e., rectangular), it is possible to uncouple the flexibility matrix along the two principal axes ( $x$ and $y$ ) to reduce its dimension. This matrix is essential to calculate the displacements of the soil

$$
\{u\}=\left[F_{S}\right] \cdot\{t\}
$$

where,

$[F s]=\left\{\mathrm{G}^{m n}\right\}_{i j}$ represents the flexibility matrix of the discretized domain, which includes the terms of Green's functions; $\{u\}$ represents the harmonic displacements; and $\{\mathrm{t}\}$ represents the surface tractions.

In fact, this relation constitutes the formulated solution, in terms of the displacements of Eq. (4) that essentially requires a discretized domain. When the foundation is in place, all elements must move as a rigid body. This condition is expressed by

$$
\{u\}=[R] \cdot\{D\},
$$

where,

$\{D\}$ denotes the displacements of the foundation; and $[R]$ represents the transformation matrix of size $(N \times 1)$.

The dynamic equilibrium between the traction forces on each element and the exterior force is expressed by

$$
\{P\}=[R]^{T} \cdot\{t\}
$$

where, $\{\mathrm{P}\}$ is the exterior force.

From Eqs. (7) and (9), the compatible forces to apply on the elements are

$$
\{t\}=\left[F_{S}\right]^{-1} \cdot[R] \cdot\{t\} .
$$

Combining Eqs. (8), (9), (10), the displacements of the foundation is obtained as

$$
\{D\}=[K(\omega)]^{-1} \cdot\{P\},
$$

where

$$
[K(\omega)]=[R]^{T} \cdot\left[F_{S}\right] \cdot[R]
$$


is the impedance function of the foundation. It is customary to introduce the dimensionless frequency $a_{o}=\omega B_{x} / C s$ at the soil-foundation interface, to scale $K(\omega)$ with static-stiffness coefficient $K_{s t}(=K(\omega=0))$ and to apply the following decomposition

$$
\left[K\left(a_{o}\right)\right]=\left[K_{s t} \cdot\left(k\left(a_{o}\right)+i a_{o} . c\left(a_{o}\right)\right)\right] \cdot(1+i 2 \beta),
$$

where,

$k\left(a_{o}\right)$ denoting the dimensionless spring coefficient; $c$ denoting the dimensionless damping coefficient and $\beta$ denoting the constant hysteretic damping coefficient.

\section{Results}

The response of square rigid foundation resting on a semi-infinite viscoelastic soil over rigid bedrock is considered. The geometry and discretization are shown in Figs. 1 and 2. Due to the space limitations only the vertical, horizontal, rocking and torsion impedances functions of the massless surface foundation are considered according dynamic equivalent-linear soil behavior described through strain-compatible shear moduli and damping coefficients for a the effect of excitation amplitude of ground motion and the dimensionless frequency. For this, three seismic records (Boumerdes-Azzazga-0.1 g, El-centro-0.2 g, Boumerdes-Kedara-0.3 g) are used in this study. Such an application represents a general study that enables the analysis of the influence of different parameters, which we will present in another article later.

In this application, the considered foundation of dimension $B=B_{x} / B_{y}=1$ is subjected to unit forces $P_{x}=P_{z}=1$ and unit moments $M_{x}=M_{z}=1$ for different dimensionless frequencies $a_{0}\left(=\omega b / C_{s}\right)$. It is noted that $\omega$ is the circular frequency and $\mathrm{C}_{\mathrm{s}}$ is the shear (S)-wave velocities. The soil is discretized into 36 quadrilateral constant elements on the soil-foundation interfaces for lower-frequency case, and it is discretized into 64 quadrilateral constant elements on the soil-foundation interfaces for the higher-frequency case.

The soil is characterised by $\rho=1, G=1, v=0.333$, and $\beta=0.05$ for linear case. In the non-linear case, the dependence of shear modulus and hysteretic damping ratio on the level of strain for the soil is described according to the established curves by (Sbartai and Filali, 2012) Fig. 3. For this, the dimensionless equivalent linear impedances functions (vertical, horizontal, rocking and torsion) of surface foundation placed on a homogeneous semi-infinite soil have been studied for different relative frequency $a_{0}$. All the results are compared with the linear case to appreciate the difference between the both cases.

Figures 3(a) and (b) show the dimensionless real part $K_{v} / K v_{\text {linear-static }}$ and dimensionless imaginary part $C_{v} / K v_{\text {linear-static }}$ of the vertical impedance as functions of the dimensionless frequency $a_{o}$ and three Earthquake records. Figure 3(a) show that the vertical dynamic stiffness coefficient naturally decreases with increasing excitation level. We remarked also of the negative values for the higher excitation level from the cutoff frequency $\mathrm{a}_{\mathrm{o}}=3$ and 2 for the second $(0.2 \mathrm{~g})$ and third earthquake $(0.3 \mathrm{~g})$ record respectively. However in Fig. 3(b), the vertical damping coefficient increases with increasing excitation level. In the equivalent-linear case, the magnitude of the 
(a)

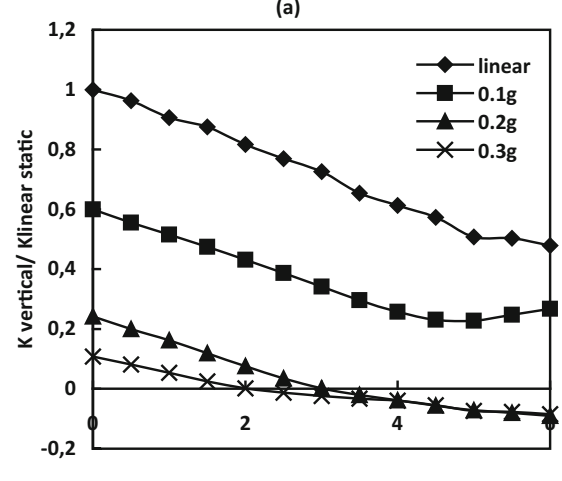

a0 (b)

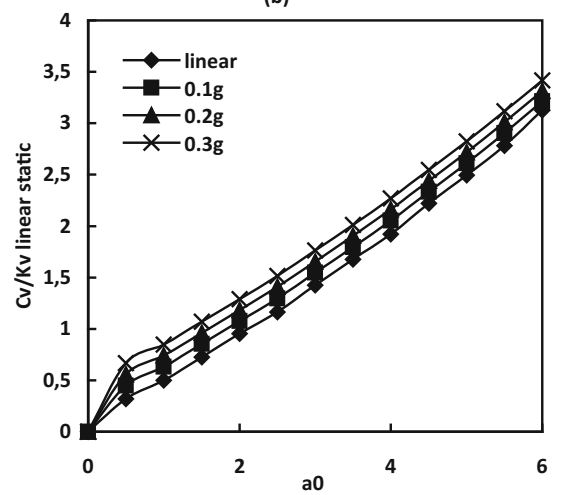

Fig. 3. Equivalent-linear vertical dynamic impedance: (a) real part (b) imaginary part

(a)

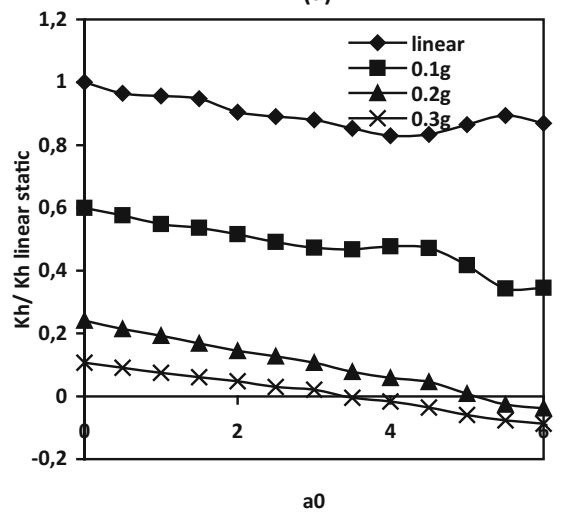

(b)

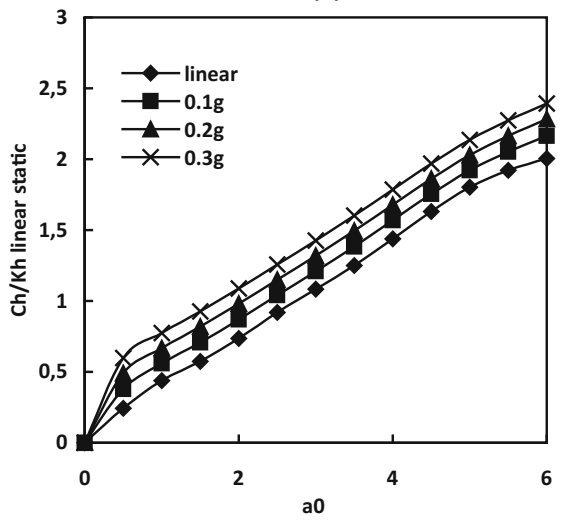

Fig. 4. Equivalent-linear horizontal dynamic impedance: (a) real part (b) imaginary part

imaginary part of the impedance increases from the linear case with increasing excitation amplitude. The higher the excitation amplitude, the larger the shift of the curve to higher values.

Figures 4(a) and (b) show the dimensionless real part $K_{h} / K h_{\text {linear-static }}$ and dimensionless imaginary part $C h / K h_{\text {linear-static }}$ of the horizontall impedance as functions of the dimensionless frequency $a_{o}$ and three Earthquake records. Figure 4(a) show that the horizontal dynamic stiffness coefficient naturally decreases with increasing excitation level. We remarked also of the negative values for the higher excitation level from the cutoff frequency $a_{o}=5$ and 4 for the second and third earthquake record respectively. In Fig. 4(b), the horizontal damping coefficient increases with increasing excitation level. In the equivalent-linear case, the magnitude of the imaginary part of the impedance increases from the linear case with increasing excitation amplitude. The higher the excitation amplitude, the larger the shift of the curve to higher values. 
(a)

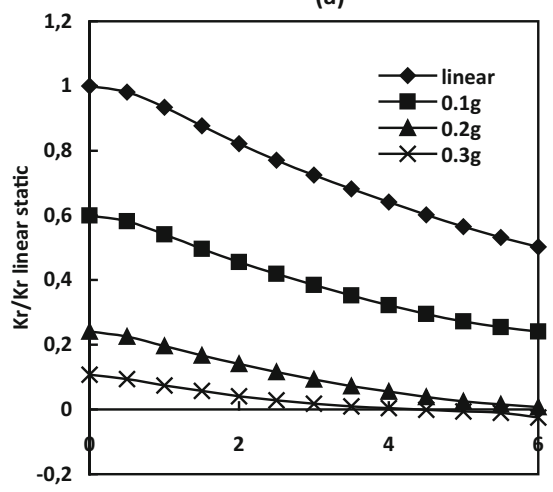

a0

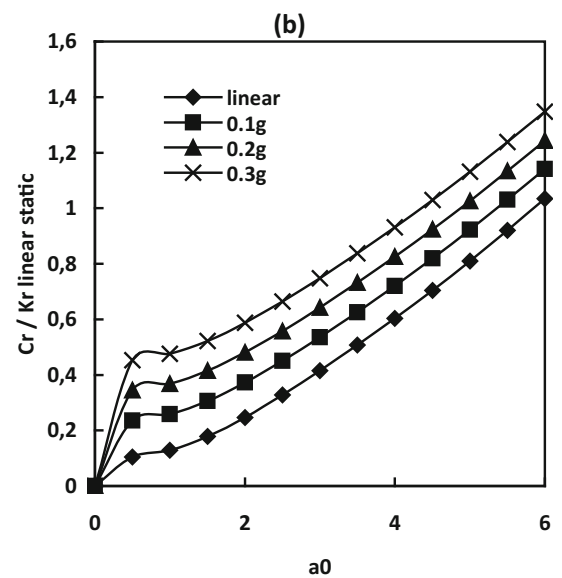

Fig. 5. Equivalent-linear rocking dynamic Impedance: (a) real part (b) imaginary part

(a)

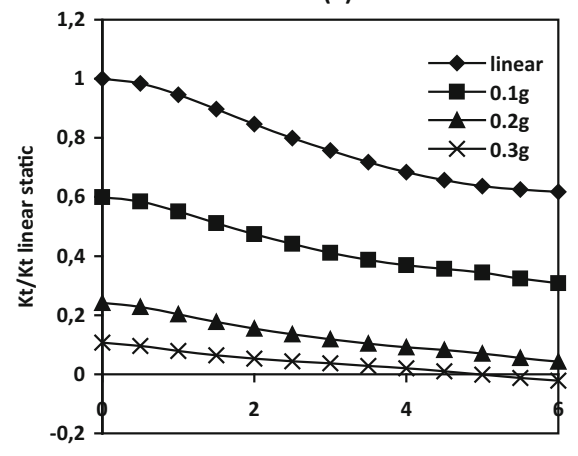

a0 (b)

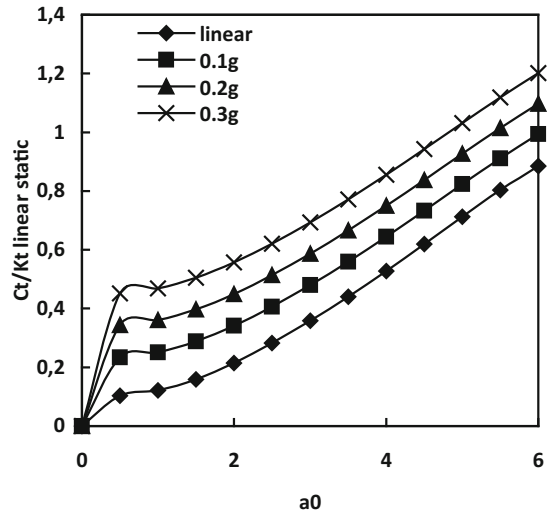

Fig. 6. Equivalent-linear torsion dynamic Impedance: (a) real part (b) imaginary part

Figures 5(a) and (b) show the dimensionless real part $K_{r} / K r_{\text {linear-static }}$ and dimensionless imaginary part $\mathrm{Cr} / \mathrm{Kh}$ linear-static of the rocking impedance as functions of the dimensionless frequency $a_{o}$ and three Earthquake records. Figure 5(a) show that the real part of the rocking impedance function decreases with increasing excitation level. We remarked also of low negative values from the cutoff frequency $a_{o}=5$ for the third earthquake record $(0.3 \mathrm{~g})$. In Fig. $5(\mathrm{~b})$, the rocking damping coefficient increases with increasing excitation level. In the equivalent-linear case, the magnitude of the imaginary part of the impedance increases from the linear case with increasing excitation amplitude. The higher the excitation amplitude, the larger the shift of the curve to higher values. 
Figures 6(a) and (b) show the dimensionless real part $K_{t} / K t_{\text {linear-static }}$ and dimensionless imaginary part $C t / K t_{\text {linear-static }}$ of the torsion impedance as functions of the dimensionless frequency $a_{o}$ and three Earthquake records. Figure 6(a) show that the real part of the torsion impedance function decreases with increasing excitation level. We remarked also of low negative values from the cutoff frequency $a_{o}=5.5$ for the third earthquake record ( $0.3 \mathrm{~g})$. In Fig. 6(b), the torsionl damping coefficient increases with increasing excitation level. In the equivalent-linear case, the magnitude of the imaginary part of the impedance increases from the linear case with increasing excitation amplitude. The higher the excitation amplitude, the larger the shift of the curve to higher values.

\section{Conclusions}

In this paper, the role of the intensity of an earthquake on the estimation of dynamic impedance functions of surface rigid foundation were study. For various seismic shaking we will have different nonlinear behavior of the soil, which may have significant effects on the amplitude and shape of the dynamic stiffness and damping coefficient. The dynamic impedance functions are estimated using an equivalent linear process and compared relative to the linear elastic case. The solution has been formulated by employing the frequency-domain boundary-element method (BEM) in conjunction with the Kausel-Peek Green's function for a layered stratum, along with a quadrilateral constant element determined using the thin-layer method (TLM). The study shows well the great importance of the loss of soil stiffness under strong shaking which proves to be more complicated than the linear elastic behavior of the soil. On the basis of the results presented in this paper, the following conclusions can be stated:

1. The nonlinear dynamic response of the foundation depends on more parameters than in the linear case:

- shear modulus reduction and damping increase curves; and

- excitation amplitude and frequency content.

2. The dynamic stiffness coefficients decrease with increasing excitation amplitude.

3. The dynamic stiffness coefficients become negative for translational modes from specific frequency $a_{o}(=2-5)$. The same case was observed for rotational modes but with a very low values and a shift of the cutoff frequency towards high frequencies $a_{o}(=5.5-6)$. It may create a detachment of the soil foundation.

4. The damping coefficients are fairly dependent on acceleration maximal of the earthquake. It increases from the linear case with increasing level of shear strain, as expected due to the increase in hysteretic soil material damping.

Acknowledgments. The authors are grateful to the Ministry of Higher Education and Scientific Research of Algeria for supporting this work. 


\section{References}

Kausel, K., et al.: Dynamic analysis of footings on layered media. J. Eng. Mech. Div. Proc. ASCE 101(EM5), 679-693 (1981)

Aubry, D., Clouteau, D.: A subdomain approach to dynamic soil-structure interaction, earthquake engineering and structural dynamics, AFPS, Nantes, pp. 251-272 (1992)

Pecker, A.: Dynamique des Sols. Presses ENPC, France (1984)

Borja, R.I., Wu, W.: Vibration of foundations on incompressible soils with no elastic regions. J. Geotech. Eng. 120(9), 1570-1592 (1994)

Sbartai, B., Boumekik, A.: Ground vibration from rigid foundation. ISET J. Technol. 45(3-4), 65-78 (2008)

Sbartai, B.: Dynamic interaction of two adjacent foundations embedded in a viscoelastic soil. Int. J. Struct. Stab. Dyn. 16(3) (2016). http://dx.doi.org/10.1142/S0219455414501107

Messioud, et.al.: Seismic response of a rigid foundation embedded in a viscoelastic soil by taking into account the soil-foundation interaction. Struct. Eng. Mech. 58(5) (2016)

Kausel, E., Peek, R.: Dynamic loads in the interior of a layered stratum: an explicit solution. Bull. Seismol. Soc. Am. 72(5), 1459-1481 (1982)

Boumekik, A.: Fonctions impédances d'une fondation vibrante en surface ou partiellement encastrée dans un sol multicouche. Ph.D. thesis, Université Libre de Bruxelles, Brussels, Belgium (1985). (in French)

Bogoliuboff, N., Mitropolsky, Y.: Asymptotic Methods in the Theory of Nonlinear Oscillations. Gordon and Breach Science Publishers, New York (1961)

Kryloff, N., Bogoliuboff, N.: Introduction to Nonlinear Mechanics. Princeton University Press, Princeton (1943). (Trans.S.L efschetz)

Seed, H.B., Idriss, I.M.: Influence of soil conditions on ground motions during earthquakes. ASCE J. Soil Mech. Found. Div. 95, 99-137 (1969)

Sbartai, B., Filali, K.: CALDYNASOIL: Software of seismic response and liquefaction potential of a soil deposit. In: 15th World Conference on Earthquake Engineering, 24 to 28 September, Lisbon, Portugal (2012) 